\title{
Alternatives To Pearson's and Spearman's Correlation Coefficients
}

Florentin Smarandache

Chair of Math \& Sciences Department

University of New Mexico

Gallup, NM 87301, USA

Abstract. This article presents several alternatives to Pearson's correlation coefficient and many examples. In the samples where the rank in a discrete variable counts more than the variable values, the mixture of Pearson's and Spearman's gives a better result.

\section{Introduction}

Let's consider a bivariate sample, which consists of $n \geq 2$ pairs $(x, y)$. We denote these pairs by:

$$
\left(\mathrm{x}_{1}, \mathrm{y}_{1}\right),\left(\mathrm{x}_{2}, \mathrm{y}_{2}\right), \ldots,\left(\mathrm{x}_{\mathrm{n}}, \mathrm{y}_{\mathrm{n}}\right)
$$

where $\mathrm{x}_{\boldsymbol{i}}=$ the value of $\mathrm{x}$ for the $\boldsymbol{i}$-th observation, and $\mathrm{y}_{i}=$ the value of $\mathrm{y}$ for the $i$-th observation, for any $1 \leq \boldsymbol{i} \leq \mathrm{n}$.

We can construct a scatter plot in order to detect any relationship between variables $\mathrm{x}$ and $\mathrm{y}$, drawing a horizontal $\mathrm{x}$-axis and a vertical $\mathrm{y}$-axis, and plotting points of coordinates $\left(\mathrm{x}_{i}, \mathrm{y}_{i}\right)$ for all $i \in\{1,2, \ldots, \mathrm{n}\}$.

We use the standard statistics notations, mostly used in regression analysis:

$$
\begin{aligned}
& \sum x=\sum_{i=1}^{n} x_{i}, \quad \sum y=\sum_{i=1}^{n} y_{i}, \quad \sum x y=\sum_{i=1}^{n}\left(x_{i} y_{i}\right), \\
& \sum x^{2}=\sum_{i=1}^{n} x_{i}^{2}, \quad \sum y^{2}=\sum_{i=1}^{n} y_{i}^{2},
\end{aligned}
$$

$\bar{X}=\frac{\sum_{i=1}^{n} x_{i}}{n}=$ the mean of sample variable $\mathrm{x}$,

$\bar{Y}=\frac{\sum_{i=1}^{n} y_{i}}{n}=$ the mean of sample variable $y$

Let's introduce a notation for the median: 


$$
\begin{aligned}
& X_{M}=\text { the median of sample variable } \mathrm{x}, \\
& Y_{M}=\text { the median of sample variable } \mathrm{y} \text {. }
\end{aligned}
$$

\section{Correlation Coefficients.}

Correlation coefficient of variables $\mathrm{x}$ and $\mathrm{y}$ shows how strongly the values of these variables are related to one another. It is denoted by $\mathrm{r}$ and $\mathrm{r} \in[-1,1]$.

If the correlation coefficient is positive, then both variables are simultaneously increasing (or simultaneously decreasing).

If the correlation coefficient is negative, then when one variable increases while the other decreases, and reciprocally.

Therefore, the correlation coefficient measures the degree of line association between two variables.

We have strong relationship if $\mathrm{r} \in[0.8,1]$ or $\mathrm{r} \in[-1,-0.8]$;

moderate relationship if $\mathrm{r} \in(0.5,0.8)$ or $\mathrm{r} \in(-0.8,-0.5)$;

And weak relationship if $\mathrm{r} \in[-0.5,0.5]$.

Correlation coefficient does not depend on the measurement unit, neither on the order of variables: $(\mathrm{x}, \mathrm{y})$ or $(\mathrm{y}, \mathrm{x})$.

If $\mathrm{r}=1$ or -1 , then there is a perfectly linear relationship between $\mathrm{x}$ and $\mathrm{y}$. If $\mathrm{r}=0$, or close to zero, then there is not a strong linear relationship, but there might be a strong non-_linear relationship that can be checked on the scatter plot.

The coefficient of determination, denoted by $\mathrm{r}^{2}$, represents the proportion of variation in $y$ due to a linear relationship between $\mathrm{x}$ and $\mathrm{y}$ in the sample:

$$
\mathrm{r}^{2}=\frac{\text { SSTo }- \text { SS Re } \text { sid }}{\text { SSTo }}=1-\frac{\text { SSRe sid }}{\text { SSTo }}
$$

where $\mathrm{SSTo}=$ total sum of squares $=\sum(y-\bar{y})^{2}=\sum_{i=1}^{n}\left(y_{i}-\bar{y}\right)^{2}$

and SSResid $=$ residual sum of squares $=\sum(y-\hat{y})^{2}=\sum_{i=1}^{n}\left(y_{i}-\hat{y}_{i}\right)$

with $\hat{y}_{i}=$ the $\mathrm{i}$-th predicted value $=\mathrm{a}+\mathrm{bx}_{\boldsymbol{i}}$ for $\boldsymbol{i} \in\{1,2, \ldots, \mathrm{n}\}$

resulting from substituting each sample $\mathrm{x}$ value into the equation for the least-squares line 
$\hat{y}=a+b x$

$$
\text { where } \mathrm{b}=\frac{\sum x y-\left[\left(\sum x\right)\left(\sum y\right) / n\right]}{\sum x^{\wedge} 2-\left[\left(\sum x\right)^{\wedge} 2 / n\right]}
$$

$$
\text { and } \mathrm{a}=\bar{Y}-\mathrm{b} \bar{X} \text {. }
$$

Obviously: coefficient of determination $=(\text { correlation coefficient })^{2}$.

Two sample correlation coefficients are well-known:

1) Pearson's sample correlation coefficient, let's denote it by $r_{p}$

$$
\mathrm{r}_{\mathrm{p}}=\frac{\sum x y-\left[\left(\sum x\right)\left(\sum y\right) / n\right]}{\sqrt{\sum x^{\wedge} 2-\left[\left(\sum x\right)^{\wedge} 2 / n\right]} \cdot \sqrt{\sum y^{\wedge} 2-\left[\left(\sum y\right)^{\wedge} 2 / n\right]}}
$$

which is the most popular;

and 2) Spearman's rank correlation coefficient, let's denote it by $r_{5}$, which is obtained from the previous one by replacing, for each $i \in\{1,2, \ldots, n\}, x_{i}$ by its rank in the variable $\mathrm{x}$, and similarly for $\mathrm{y}_{i}$.

We propose more alternative sample correlation coefficients in the following ways, replacing in Pearson's formula (9):

3.1. Each $\mathrm{x}_{i}$ by its deviation from the $\mathrm{x}$ mean: $\mathrm{x}_{i}-\bar{x}$, and each $\mathrm{y}_{i}$ by its deviation from the y mean: $\mathrm{y}_{i}-\bar{y}$.

3.2. Each $\mathrm{x}_{i}$ by its deviation from the $\mathrm{x}$ minimum: $\mathrm{x}_{\boldsymbol{i}}-\mathrm{x}_{\mathrm{min}}$, and each $\mathrm{y}_{\boldsymbol{i}}$ by its deviation from the y minimum: $y_{i}-y_{\min }$.

3.3. Each $\mathrm{x}_{i}$ by its deviation from the $\mathrm{x}$ maximum: $\mathrm{x}_{\max }-\mathrm{x}_{i}$, and each $\mathrm{y}_{i}$ by its deviation from the $y$ maximum: $y_{\max }-y_{i}$

3.4. Each $\mathrm{x}_{\boldsymbol{i}}$ by its deviation from a given $\mathrm{x}_{\mathrm{k}}($ for $\mathrm{k} \in\{1,2, \ldots, \mathrm{n}\})$ :

$$
\mathrm{X}_{\mathrm{i}}-\mathrm{X}_{\mathrm{k}}
$$

and each $\mathrm{y}_{i}$ by its deviation from the corresponding given $\mathrm{y}_{k}$ :

$$
\mathrm{y}_{\mathrm{i}}-\mathrm{y}_{\mathrm{k}}
$$


Not surprisingly, all these four alternative sample correlation coefficients are equal to Pearson's since they are simply related to translations of Cartesian axes, whose origin $(0,0)$ is moved to $(\bar{x}, \bar{y}),\left(\mathrm{x}_{\min }, \mathrm{y}_{\min }\right),\left(\mathrm{x}_{\max }, \mathrm{y}_{\max }\right)$, or $\left(\mathrm{x}_{\mathrm{k}}, \mathrm{y}_{\mathrm{k}}\right)$ respectively.

Example: Let the variables $\mathrm{x}, \mathrm{y}$ be given below:

\begin{tabular}{l|r|r|r|r|r|r|r|r|r|r|}
$\mathrm{x}$ & 6 & 7 & 12 & 14 & 23 & 41 & 53 & 60 & 69 & 72 \\
\hline $\mathrm{y}$ & 2.5 & 1.1 & 6.3 & 2.1 & 2.9 & 15.3 & 20.7 & 18.4 & 22 & 33
\end{tabular}

$\underline{\text { Table } 1}$

and their scatter plot:

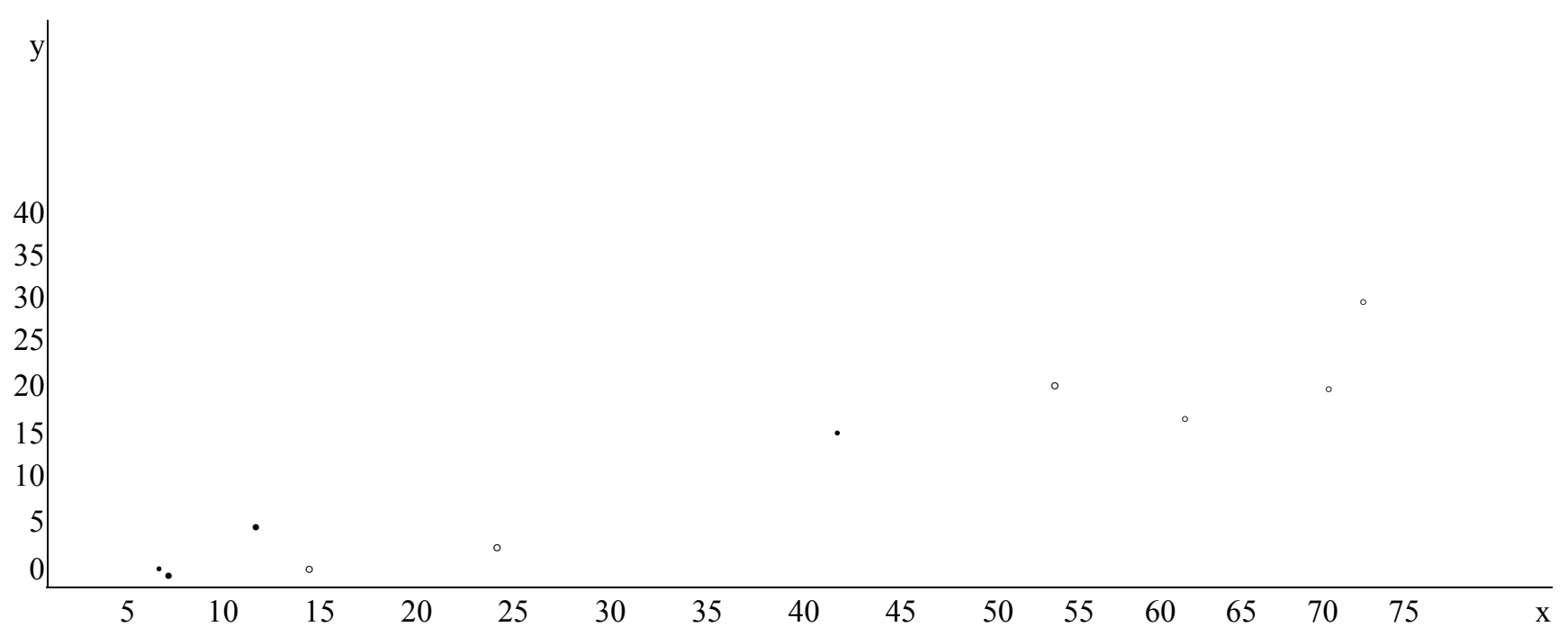

\section{Graph 1}

1) Calculating Pearson's correlation coefficient:

$$
\begin{array}{ll}
\sum x=357 ; & \bar{x}=35.7 ; \\
\sum y=124.3 ; & \bar{y}=12.43 ; \\
\sum x^{2}=18,989 ; & \\
\sum y^{2}=2,634.11 ; & \\
\sum x y=6,916.8 ; &
\end{array}
$$




$$
r_{p}=0.95075
$$

2) Calculating Spearman's rank correlation coefficient:

\begin{tabular}{l|l|l|l|l|l|l|l|l|r|r|}
\hline $\mathrm{x}$ & 1 & 2 & 3 & 4 & 5 & 6 & 7 & 8 & 9 & 10 \\
\hline $\mathrm{y}$ & 3 & 1 & 5 & 2 & 4 & 6 & 8 & 7 & 9 & 10
\end{tabular}

\section{$\underline{\text { Table } 2}$}

$$
\begin{aligned}
& \sum x=\frac{(1+10) \cdot 10}{2}=11.5=5.5 ; \\
& \sum y=55 \\
& \sum x^{2}=385 ; \\
& \sum y^{2}=385 ; \\
& \sum x y=377 \\
& \mathrm{r}_{\mathrm{s}}=0.90303 .
\end{aligned}
$$

3.1) Replacing $\mathrm{x}_{i}$ by $\mathrm{x}_{i}-\bar{x}$ and $\mathrm{y}_{i}$ by $\mathrm{y}_{i}-\bar{y}$ for all $\boldsymbol{i}$ (deviations from the mean):

\begin{tabular}{l|r|r|r|r|r|r|r|r|r|r|}
$\mathrm{x}$ & -29.7 & -28.7 & -23.7 & -21.7 & -12.7 & 5.3 & 17.3 & 24.3 & 33.3 & 36.3 \\
\hline $\mathrm{y}$ & -9.93 & -11.33 & -6.13 & -10.33 & -9.53 & 2.87 & 8.27 & 5.97 & 9.57 & 20.57
\end{tabular}

\section{$\underline{\text { Table } 3}$}

Similarly: $\quad \sum x=0$,

$$
\begin{gathered}
\text { because } \sum x=\sum_{i=1}^{10}\left(x_{i}-\bar{x}\right)=\mathrm{x}_{1}-\bar{x}+\mathrm{x}_{2}-\bar{x}+\ldots+\mathrm{x}_{10}-\bar{x}=\left(\mathrm{x}_{1}+\mathrm{x}_{2}+\ldots+\mathrm{x}_{10}\right)-10 \bar{x} \\
=\left(\mathrm{x}_{1}+\mathrm{x}_{2}+\ldots+\mathrm{x}_{10}\right)-10 \cdot \frac{x_{1}+x_{2}+\ldots+x_{n}}{10}=0 \\
\sum y=0 \\
\sum x^{2}=6,244.10 \\
\sum y^{2}=1,089.06
\end{gathered}
$$




$$
\begin{gathered}
\sum x y=2,479.29 \\
r_{\text {mean }}=0.95075
\end{gathered}
$$

3.2) Replacing $x_{i}, y_{i}$ by their deviations from the smaller $\mathrm{x}$ : $=\mathrm{x}-\mathrm{x}_{\text {small }}$ and $\mathrm{y}:=\mathrm{y}-\mathrm{y}_{\text {small }}$ we have a translation of axes again.

\begin{tabular}{l|r|r|r|r|r|r|r|r|r|r|}
$\mathrm{x}$ & 0 & 1 & 6 & 8 & 17 & 35 & 47 & 54 & 63 & 66 \\
\hline $\mathrm{y}$ & 1.4 & 0 & 5.2 & 1 & 1.8 & 14.2 & 19.6 & 17.3 & 20.9 & 31.9
\end{tabular}

\section{$\underline{\text { Table } 4}$}

$$
\begin{aligned}
& \sum x=297 ; \\
& \sum y=113.3 ; \\
& \sum x^{2}=15,065 ; \\
& \sum y^{2}=2,372.75 ; \\
& \sum x y=5,844.30 ; \\
& \mathrm{r}_{(\text {small })}=0.95075 .
\end{aligned}
$$

3.3) Replacing $\mathrm{x}_{i}, \mathrm{y}_{i}$ by their deviations from the maximum:

\begin{tabular}{l|r|r|r|r|r|r|r|r|r|r|}
$\mathrm{x}$ & 66 & 65 & 60 & 58 & 49 & 31 & 19 & 12 & 3 & 0 \\
\hline $\mathrm{y}$ & 30.5 & 31.9 & 26.7 & 30.9 & 30.1 & 17.7 & 12.3 & 14.6 & 11 & 0
\end{tabular}

$\underline{\text { Table } 5}$

$$
\begin{aligned}
& \sum x=363 ; \\
& \sum y=205.7 ; \\
& \sum x^{2}=19,421 ; \\
& \sum y^{2}=5,320.31 ; \\
& \sum x y=9,946.20 ; \\
& r_{(\max )}=0.95075 .
\end{aligned}
$$

3.4) Replacing $\mathrm{x}_{i}$ by $\mathrm{x}_{i}-\mathrm{x}_{4}$ and $\mathrm{y}_{i}$ by $\mathrm{y}_{i}-\mathrm{y}_{4}$ (in this case $\left.\mathrm{k}=4\right),\left(\mathrm{x}_{4}, \mathrm{y}_{4}\right)=(14,2.1)$ :

\begin{tabular}{l|r|r|r|r|r|r|r|r|r|r|}
$\mathrm{x}$ & -8 & -7 & -2 & 0 & 9 & 27 & 39 & 46 & 55 & 58 \\
\hline $\mathrm{y}$ & 0.4 & -1 & 4.2 & 0 & 0.8 & 13.2 & 18.6 & 16.3 & 19.9 & 30.9
\end{tabular}

$\underline{\text { Table } 6}$

$$
\begin{aligned}
& \sum x=217 \\
& \sum y=103.3
\end{aligned}
$$




$$
\begin{aligned}
& \sum x^{2}=10,953 \\
& \sum y^{2}=2,156.15 \\
& \sum x y=4,720.9 \\
& \mathrm{r}_{4}=\mathrm{r}_{i}=0.95075 \text { for any } \boldsymbol{i} \in\{1,2, \ldots, 10\} .
\end{aligned}
$$

Similarly if we replace in Pearson's formula (9) and also getting the same result equals to $\mathrm{r}_{\mathrm{p}}$ :

3.5) Each $x_{i}$ by its deviation from $x$ 's median, and each $y_{i}$ by its deviation from $y$ 's median.

3.6) Each $x_{i}$ by its deviation from $x$ 's standard deviation, and each $y_{i}$ by its deviation from y's standard deviation.

3.7) Each $x_{i}$ by $x_{i} \pm a$ (where a is any number), and each $y_{i}$ by $y_{i} \pm b$ (where $b$ is any number).

3.8) Each $\mathrm{x}_{i}$ by $\mathrm{x}_{i} * \mathrm{a}$ (where a is any non-zero number and “*” is either division or multiplication), and each $\mathrm{y}_{i}$ by $\mathrm{y}_{i}{ }^{*} \mathrm{~b}$ (similarly for $\mathrm{b}$ and “*”).

Since the cases $3.5-3.7$ are similar to $3.1-3.4$, let's consider two examples for the case 3.8:

3.8.1) Suppose each $x_{i}$ in the original example, Table 1 , is divided by 5 , while each $y_{i}$ is divided by 2 .

Then:

$$
\begin{aligned}
& \sum x=71.4 \\
& \sum y=62.15 \\
& \sum x^{2}=759.56 \\
& \sum y^{2}=658.528 \\
& \sum x y=691.68 \\
& \mathrm{r}_{\text {(division, division) }}=0.95075 .
\end{aligned}
$$

3.8.2) Now, let's still divide each $x_{i}$ in Table 1 by 5 , but this time multiply each $y_{i}$ with 2.

$$
\text { Then: } \begin{aligned}
\sum x & =71.4 ; \\
\sum y & =248.6
\end{aligned}
$$




$$
\begin{aligned}
& \sum x^{2}=759.56 \\
& \sum y^{2}=10,536.4 \\
& \sum x y=2,766.72
\end{aligned}
$$

$\mathrm{r}_{(\text {division, multiplication })}=0.95075$.

So, again these results coincide with Pearson's.

More interesting alternative correlation coefficients [and given different results from Pearson's and Spearman's] are obtained by doing:

\section{A mixture of Pearson's and Spearman's correlation coefficients.}

4.1 We only replace $x_{i}$ by its rank among $x^{\prime}$ 's, while $y_{i}$ remains unchanged:

\begin{tabular}{l|r|r|r|r|r|r|r|r|r|r|}
$\mathrm{x}$ rank & 1 & 2 & 3 & 4 & 5 & 6 & 7 & 8 & 9 & 10 \\
\hline $\mathrm{y}$ & 2.5 & 1.1 & 6.3 & 2.1 & 2.9 & 15.3 & 20.7 & 18.4 & 22 & 33
\end{tabular}

Table 7

$$
\begin{aligned}
& \sum x=55 \\
& \sum y=124.3 \\
& \sum x^{2}=385 \\
& \sum y^{2}=2,634.11 \\
& \sum x y=958.4 \\
& \mathrm{r}_{\mathrm{s}, \mathrm{p}}=0.91661 \in[0.90303,0.95075] .
\end{aligned}
$$

4.2. Similarly, as above, let's only replace $\mathrm{y}_{i}$ by its rank among $\mathrm{y}$ 's, while $\mathrm{x}_{i}$ remains unchanged.

\begin{tabular}{l|r|r|r|r|r|r|r|r|r|r|}
$\mathrm{x}$ & 6 & 7 & 12 & 14 & 23 & 41 & 53 & 60 & 69 & 72 \\
\hline $\mathrm{y}$ rank & 3 & 1 & 5 & 2 & 4 & 6 & 8 & 7 & 9 & 10
\end{tabular}

$\underline{\text { Table } 8}$

$$
\begin{aligned}
& \sum x=357 \\
& \sum y=55 \\
& \sum x^{2}=18,989 \\
& \sum y^{2}=385 \\
& \sum x y=2,636 \\
& \mathrm{r}_{\mathrm{p}, \mathrm{s}}=0.93698 \in[0.90303,0.95075] .
\end{aligned}
$$


Both mixture correlation coefficients give different results from Pearson's and Spearman's, actually they are in between.

\section{Conclusion:}

In the samples where the rank in a discrete variable counts more than the variable values, this mixture of correlation coefficients brings better results than Pearson's or Spearman's.

\section{Reference:}

Jay Devore, Roxy Peck, "Introductory Statistics”, second edition, West Publ. Co., 1994. 